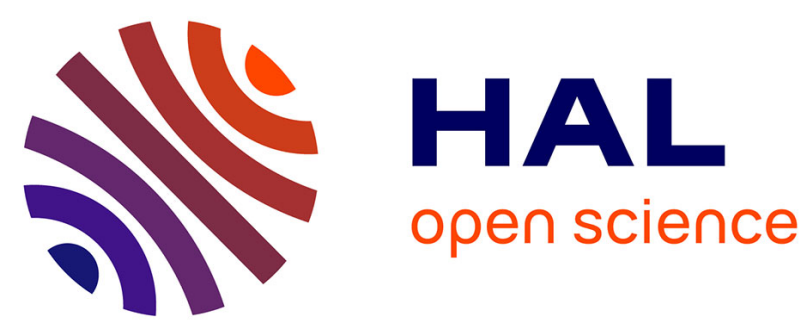

\title{
Making Prussian blue analogues nanoparticles luminescent: effect of the luminophore confinement over the properties
}

Ekaterina Mamontova, Jérôme Long, Rute A.S. Ferreira, Alexandre Botas, Fabrice Salles, Yannick Guari, Luís D. Carlos, Joulia Larionova

\section{To cite this version:}

Ekaterina Mamontova, Jérôme Long, Rute A.S. Ferreira, Alexandre Botas, Fabrice Salles, et al.. Making Prussian blue analogues nanoparticles luminescent: effect of the luminophore confinement over the properties. Nanoscale, 2019, 11, pp.7097. 10.1039/C8NR09982K . hal-02096496

\section{HAL Id: hal-02096496 https://hal.science/hal-02096496}

Submitted on 26 Nov 2020

HAL is a multi-disciplinary open access archive for the deposit and dissemination of scientific research documents, whether they are published or not. The documents may come from teaching and research institutions in France or abroad, or from public or private research centers.
L'archive ouverte pluridisciplinaire HAL, est destinée au dépôt et à la diffusion de documents scientifiques de niveau recherche, publiés ou non, émanant des établissements d'enseignement et de recherche français ou étrangers, des laboratoires publics ou privés. 


\title{
Making Prussian blue analogues nanoparticles luminescent: effect of the luminophore confinement over the properties.
}

\author{
Ekaterina Mamontova, ${ }^{a}$ Jérôme Long*, ${ }^{a}$ Rute A. S. Ferreira, ${ }^{b}$ Alexandre M. P. Botas, ${ }^{b}$ Fabrice \\ Salles, ${ }^{d}$ Yannick Guari, ${ }^{a}$ Luis D. Carlos ${ }^{b}$ and Joulia Larionova ${ }^{a}$
}

We report in this communication the post-synthetic functionalization of $\mathrm{K}^{+} / \mathrm{Ni}^{2+} /\left[\mathrm{Cr}(\mathrm{CN})_{6}\right]^{3-}$ Prussian Blue Analogue (PBA) nanoparticles by the 2-aminoanthracene luminophore to yield a bifunctional magneto-luminescent nanosystem. The photoluminescence properties of the fluorophore are found modified by confinement effet upon adsorsption, while the magnetic behavior of PBA is preserved.

Multifunctional molecular materials are systems combining several physical or chemical properties. ${ }^{1}$ Such smart systems have attracted a great deal of attention not only from a fundamental point of view, but also due to their future technological applications. During the last two decades, advances have been made by combining magnetic properties with optical or nonlinear activity, ${ }^{2}$ conductivity, ${ }^{3}$ porosity, ${ }^{4}$ electron switching or ferroelectricity. ${ }^{5-6}$ Moreover and despite the frequent emission quenching activated by paramagnetic transitions metal ions, many efforts have been recently devoted to the synthesis of molecule-based magnets exhibiting photoluminescence. ${ }^{7-9}$

Another aspect of multifunctional molecular materials, which has attracted a high interest in the recent decade, consists in their controlling design at the nanoscale. In this line of thought, two families of multifunctional molecular materials, well known as Metal-Organic Frameworks (MOF), ${ }^{10}$, 11 and Prussian Blue Analogues (PBA) nano-objects, have been developed. The latter constitutes an exciting class of inorganic nanosystems ${ }^{12-13}$ made from transition metal ions assembled by cyano ligands into a 3D porous structure of general formula $A_{x} M\left[M^{\prime}(C N)_{6}\right]_{y} \square_{z}$ (A is a monovalent cation, $M$ and $M^{\prime}$ are transition metal ions, $\square$ represents the cyanometallate vacancies). In addition to the advantages relative to their molecular nature, such as optical transparency, low density or soft synthetic routes; they present numerous assets: (i) a versatility of chemical compositions giving rise to various properties (magnetism, optics, conductivity) that may be adjusted by the careful choice of the metal ions; (ii) a porous structure that may be used to encapsulate various species; (iii) their chemical robustness towards various media making them particularly adapted for future applications.

Surprisingly, although efforts have been conducted to render these nanosized PBA multifunctional, mainly for their possible use in various applications, ${ }^{13}$ combination with luminescent probes remains almost unexplored. The association of magnetism and luminescence in nanosized PBA could be highly interesting both from a fundamental point of view to study the synergy between the two properties as for potential applications in sensors, biology and electronics to name a few. To our knowledge, the only examples reported in the literature consist in Mn-doped Prussian Blue (PB) nanoparticles whose surface was functionalized with the fluorescent avidin or core@shell made of polypyrrole@PB nanoparticles. ${ }^{14-15}$ On the other hand, while the adsorption of guest luminophores is one of the most powerful strategies to render MOF luminescent, ${ }^{16-}$ ${ }^{19}$ it has never been applied to PBA systems. In this sense, we report here the post-synthetic functionalization of magnetic $\mathrm{K}^{+} / \mathrm{Ni}^{2+} /\left[\mathrm{Cr}(\mathrm{CN})_{6}\right]^{3-}$ PBA nanoparticles by a 2-aminoanthracene (AA) luminophore to yield a bifunctional magneto-luminescent nanosystem. Both, magnetic and photoluminescent properties were investigated and combined with molecular simulations to determine the adsorption mechanism.

Our strategy to implement luminescence in magnetic PBA nanoparticles takes advantage of the great flexibility of this coordination framework and uses a two-step approach consisting in the synthesis of the surface free $\mathrm{K}^{+} / \mathrm{Ni}^{2+} /\left[\mathrm{Cr}(\mathrm{CN})_{6}\right]^{3-}$ nanoparticles and their post-synthetic functionalization with AA. This PBA was chosen owing to its interesting magnetic properties (a relatively high Curie temperature around $65 \mathrm{~K}$ ) and the absence of absorption bands in the visible region of the electronic spectrum to avoid the quenching of the guest's luminescence. On the other hand, the AA luminophore was selected due to: $i$ ) the presence of a donor amine function that allows its anchorage via the formation of hydrogen or coordination bonds to the $\mathrm{Ni}^{2+}$ metal ions located close to the cyanometallate vacancies; ii) its suitable molecular size that can fit into cavities generated by the hexacyanochromate vacancies; iii) its green colour emission upon various excitation wavelengths ranging from 300 to $450 \mathrm{~nm},{ }^{20}$ which makes it widely used in applications, such as reagents and assay kits.

Firstly, $\mathrm{K}_{0.04} \mathrm{Ni}\left[\mathrm{Cr}(\mathrm{CN})_{6}\right]_{0.64} \cdot 4.1 \mathrm{H}_{2} \mathrm{O}$ (1) PBA nanoparticles of 65 $\mathrm{nm}$ were obtained in aqueous solution by the controlled addition of the respective molecular precursors, $\mathrm{NiCl}_{2} \cdot 6 \mathrm{H}_{2} \mathrm{O}$ and $\mathrm{K}_{3}\left[\mathrm{Cr}(\mathrm{CN})_{6}\right]$, using the previously reported synthetic strategy for other PBA. ${ }^{21}$ Subsequently, the obtained nanoparticles were dispersed in an ethanol solution containing the $\mathbf{A A}$ luminophore and further reacted for $24 \mathrm{~h}$ to give $\mathrm{K}_{0.04} \mathrm{Ni}\left[\mathrm{Cr}(\mathrm{CN})_{6}\right]_{0.60} @(\mathrm{AA})_{0.18} \cdot 3.8 \mathrm{H}_{2} \mathrm{O}(\mathbf{1} @ \mathrm{AA})$ as a brown-yellow solid (Fig. 1b). The resulting functionalized nanoparticles were thoroughly washed with ethanol and next dialyzed to fully remove any physisorbed AA moieties.

The pristine nanoparticles 1 exhibit the characteristic structural features of the bulk PBA, namely a stretching cyanide $\mathrm{v}\left(\mathrm{Cr}{ }^{\prime \prime \prime}-\mathrm{CN}-\mathrm{Ni} \mathrm{i}^{\prime \prime}\right)$ band located at $2173 \mathrm{~cm}^{-1}$ in the FT-IR spectra (Fig. S1), as well as its Powder X-Ray Diffraction Pattern showing the typical $f c c$ structure with a lattice parameter $a=$ $10.44 \AA$ (Fig. S2). The crystallite size obtained from the Scherrer law is equal to $55 \mathrm{~nm}$ ( $d_{200}$ reflection) and is in good 
accordance with the mean diameter of $65 \pm 6 \mathrm{~nm}$ of the cubic nanoparticles determined by Transmission Electronic Microscopy (TEM) (Fig. S3). The post-synthetic functionalization of $\mathrm{K}^{+} / \mathrm{Ni}^{2+} /\left[\mathrm{Cr}^{\prime \prime \prime}(\mathrm{CN})_{6}\right]^{3-}$ giving 1@AA is confirmed by the FT-IR spectrum, which shows the typical vibrations bands of the luminophore in the $600-1700 \mathrm{~cm}^{-1}$ region (Fig. S1). Elemental and thermogravimetric (TGA) analyses (Fig. S4) allow the determination of the AA and water content giving the formula 1@AA as $\mathrm{K}_{0.04} \mathrm{Ni}\left[\mathrm{Cr}(\mathrm{CN})_{6}\right]_{0.60} @(\mathrm{AA})_{0.18} \cdot 3.8 \mathrm{H}_{2} \mathrm{O}$. This corresponds to a loading capacity (defined as the amount of AA divided by the total nanoparticle weight) of $11.9 \mathrm{wt} \%$. Since PBA incorporate both, coordinated and zeolitic water molecules, the slight difference in the water content for 1 and 1@AA may originate from either, the adsorption of $\mathbf{A A}$, as observed for $\mathrm{Cs}^{+}$ adsorption, $^{22}$ or from different number of zeolitic water molecules before and after functionalization. Yet, TGA indicates that the decomposition of the fluorophore in 1@AA occurs in the temperature range $325-400^{\circ} \mathrm{C}$, slightly greater that of the PBA $\left(300-325^{\circ} \mathrm{C}\right)$. Remarkably, this remains much higher than the decomposition temperature of $\mathbf{A A}$ at $200^{\circ} \mathrm{C}$, suggesting that in 1@AA, the luminophore AA is efficiently protected within the porosity. Additional characterisations show that: i) 1@AA retain their initial structure, shape and size (Fig. 1d, Fig. S1-S2); ii) unreacted AA crystallites are not present at the particles surface (Fig. S5); iii) 1 and 1@AA have an identical zeta-potential, which indicates a similar surface state (Table S1). All these data suggest that AA is localized within the porosity of the PBA rather than anchored at the nanoparticles' surface.

To confirm this hypothesis, molecular simulations (Monte Carlo and classical force field-based calculations) were performed (ESI). The pores size distribution (Fig. S6) shows the occurrence of two different porisity in the PBA network: tetrahedral sites of the $c f c$ structure (pore diameter close to $3.5 \AA$ ) and larger elongated pores formed by cyanometallates vacancies with dimensions of $8 \AA \times 8 \AA \times 11 \AA$, suitable to accommodate AA (10 ̊ $\times 5 \AA$ × $1 \AA$ ).



Figure 1. a) Scheme of the synthetic strategy used in the post-synthetic functionalization of PBA nanoparticles; b) Photographs of 1 and $1 @ A A ; c)$ Photographs of 1 and 1@AA under excitation with an UV lamp at 365 nm; d) TEM image of 1@AA. Inset: corresponding histogram of the size distribution. The solid line represents the fit with a Gaussian function.

Additionally, the saturation capacity obtained by Monte Carlo simulations taking the hypothesis of the AA loading in an empty structure is estimated at 4.50 AA molecules per unit cell, which is higher than the experimental value of 0.72 . This corresponds to a loading capacity of $74.4 \mathrm{wt} \%$ to compare with the experimental one of $11.9 \mathrm{wt} \%$. However, this theoretical capacity was obtained without taking into account the presence of solvent molecules, which are able to chemically interact with unsaturated metal centers (CUS) and enters in competition with AA. In this line, molecular simulations indicate that the ethanol molecules fixed on the CUS prevent the adsorption of AA. Since AA molecules are located in the large micropores formed by vacancies and distributed randomly, the observed discrepancy between theoretical and experimental capacity could be explained by diffusion issues of AA upon adsorption (Fig. S7). Moreover, in order to provide a deeper insight in the mechanism of interaction between $\mathbf{A A}$ and the PBA network, complementary calculations have been performed to estimate the interaction energy taken the hypothesis of the AA adsorption by physisorption (since Universal Force Field is unable to predict the formation of a covalent interaction). Firstly, the enthalpy corresponding to a physisorption at low coverage (1 molecule per cell) is estimated at $-21 \mathrm{kcal}^{\mathrm{mol}}{ }^{-1}$, while the chemisorption involving a coordination bond between $\mathbf{A A}$ and $\mathrm{Ni}^{2+}$ gives $-27 \mathrm{kcal}^{-\mathrm{mol}^{-1}}$. Such difference of $6 \mathrm{kcal} . \mathrm{mol}^{-1}$ in the enthalpies between the two mechanisms suggests a favourable interaction by coordinative bonds between AA and the PBA. Fig. S7 illustrates this interaction between $\mathrm{NH}_{2}$ from $\mathrm{AA}$ and $\mathrm{Ni}^{2+}$ of $2.39 \AA$ 
obtained by Monte-Carlo (physisorption), but it should be mentioned that interaction between $\mathrm{NH}_{2}$ and cyano-groups can be observed. In contrast, by considering chemisorption, a shorter distance of $1.79 \AA$ A between $\mathrm{AA}$ and $\mathrm{Ni}^{2+}$ is found.

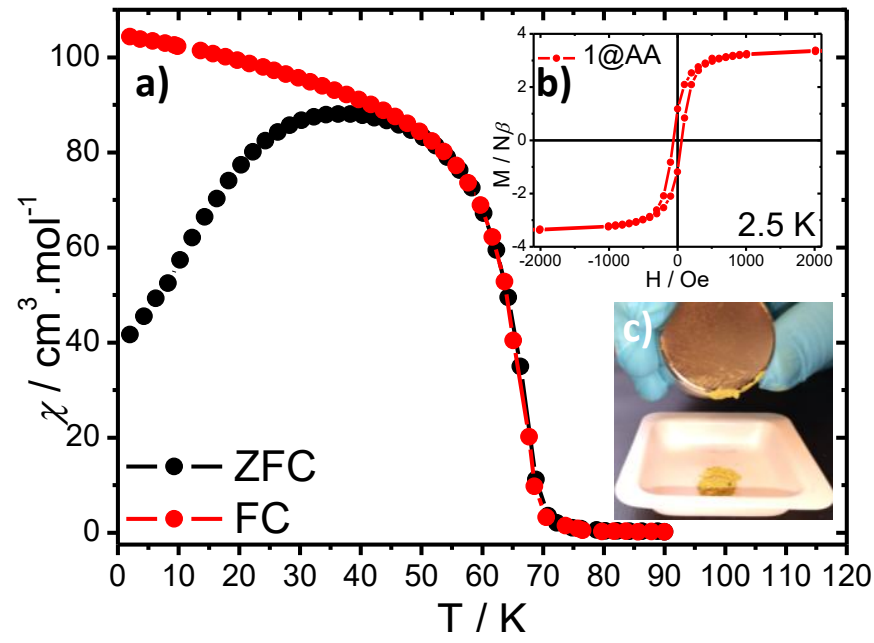

Figure 2. a) ZFC/FC curves performed for 1@AA with an applied magnetic field of 100 Oe ; b) Field dependence of the magnetization at $2.5 \mathrm{~K}$ for 1@AA; c) Photograph 1@AA interacting with a magnet at room temperature.

The magnetic properties investigations on 1@AA reveal the typical behaviour closed to the one observed for the bulk $\mathrm{K}^{+} / \mathrm{Ni}^{2+} /\left[\mathrm{Cr}^{\prime \prime \prime}(\mathrm{CN})_{6}\right]^{3-}$ PBA. It is in good accordance with the size of the considered nanoparticles, which are much larger than the single domain critical size estimated at around $22 \mathrm{~nm}^{23}$ Hence, the magnetic irreversibility was studied by applying the Zero Field-Cooled (ZFC)-Field Cooled (FC) procedure. The ZFC curve of $1 @ A A$ exhibits a peak with a maximum at $40 \mathrm{~K}$, while the FC curve increases upon cooling (Fig. 2a). From the ZFC, the Curie temperature, $T_{\mathrm{c}}$, is estimated at $65 \mathrm{~K}$, which remains unchanged upon functionalization (Fig. S8). The field dependence of the magnetization measured at $2.5 \mathrm{~K}$ shows a saturation value of $3.4 N \beta$ in accordance with the ferromagnetic interaction between $\mathrm{Ni}^{2+}$ and $\mathrm{Cr}^{3+}$ ions and a hysteresis loop with a small coercive field of 110 Oe confirming the blocking of the magnetization (Fig. 2b, Fig. S9). ${ }^{24}$ In spite of the low $T_{\mathrm{c}}$, the strong paramagnetism of 1@AA allows its interaction with a magnetic field even at room temperature as shown in Fig. 2c.

The photoluminescence of 1@AA was investigated in details. Whereas $\mathbf{1}$ is optically inactive, $\mathbf{1} @ A$ A displays a green emission under excitation by a UV-lamp (Fig. 1c). Moreover, the nanoparticles could be re-dispersed in various solvents, such as water and ethanol, retaining their absorption (Fig. S10) and emission properties (Figs. S11, S12). Apart from minor blue-shift (e.g., $\sim 5 \mathrm{~nm}$, at $300 \mathrm{~K}$ and $16 \mathrm{~K}$, Figs. 3, S13), the solid-state emission spectrum of 1@AA resembles to what is observed for AA. The broad asymmetric emission band, centred at $500 \mathrm{~nm}$, is ascribed to the AA fluorescence, ${ }^{25}$ being excited from the UV to the blue spectral regions $(290-435 \mathrm{~nm}$, Fig. 3 and Fig. S14-S15). The slight blue-shift suggests an interaction between $\mathbf{A A}$ and the coordination framework when the chromophore is adsorbed. This is reinforced by the comparison of the excitation solid-state spectra for AA and 1@AA. Indeed, whereas the excited singlet states absorption dominates the AA spectra (vertical lines, Fig. 3), ${ }^{26}$ a low-energy band at $\sim 435 \mathrm{~nm}$ is the main excitation channel of the 1@AA emission. This component, also seen in the excitation spectra of AA at $\sim 455 \mathrm{~nm}$, is strongly dependent of the chromophore's concentration, as Fig. S12 shows for AA and 1@AA in ethanolic suspensions. This dependence is a strong argument favouring the modification in the environment of AA molecules (through $\pi-\pi$ or intermolecular hydrogen bonds) upon adsorption within PBA. The possibility of this band be ascribed to a $T_{n} \leftarrow T_{1}$ absorption (where $T_{1}$ and $T_{n}$ are excited triplet states), similar to what was reported for anthracene after protonation, ${ }^{27}$ is discharged because here there is no sign of protonation.

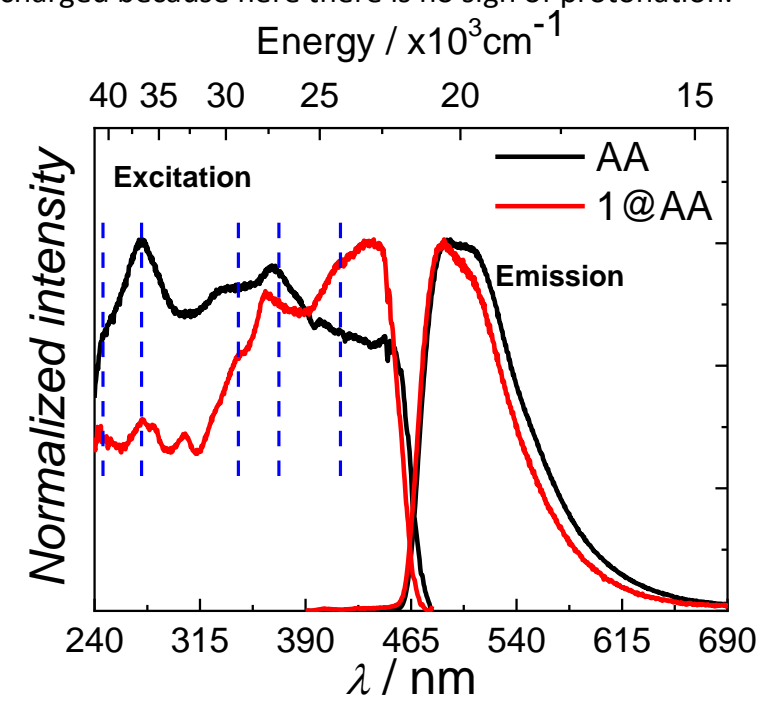

Figure 3. Low temperature (16 K) excitation and emission spectra monitored around $500 \mathrm{~nm}$ and excited at $365 \mathrm{~nm}$, respectively for powdered AA and 1@AA.

To go further in the photoluminescence characterization of 1@AA, the temperature dependence of the steady-state emission (Figs. S16-S20) and the thermal dependence of the emission lifetime (Figs. S21-S23) were studied. The emission integrated intensities of AA and 1@AA reveal a similar decrease as the temperature is raised from 16 to $300 \mathrm{~K}$ (Fig. S20), suggesting a comparable non-radiative transition probability for both samples indicating that the PBA does not induce extra-non-radiative pathways. The decay curves of both samples are well described by a single exponential function suggesting a homogeneous distribution of AA molecules. The lifetime $(\tau)$ values (in the $10^{-9} \mathrm{~s}$ timescale) are in good agreement with a singlet-related emission. Despite an analogous trend with the temperature increase, we note a larger decrease for 1@AA. Thus, at $20 \mathrm{~K}$, the value of $16.9 \pm$ $0.2 \mathrm{~ns}$ is larger than that of AA of $14.7 \pm 0.1 \mathrm{~ns}$, whereas at 300 $K$ the lifetime values decrease to $7.3 \pm 0.1$ and $8.8 \pm 0.5 \mathrm{~ns}$, for $1 @ A A$ and AA, respectively (Fig. S23). Such differences may be rationalised attending to the fact that the experimental transition probability may be expressed as $\tau^{-1}=\tau_{n r}{ }^{-1}+\tau_{r}^{-1}$, where $\tau_{n r}$ and $\tau_{r}$ represent the non-radiative and radiative lifetimes, respectively, and that at $12 \mathrm{~K}^{-1} \sim \tau_{r}^{-1}$. Thus, the greater lowtemperature $\tau$ value of 1 @AA indicates a decrease in the radiative transition probability caused by the adsorption of AA.

Nonetheless, the decrease in $\tau$ as the temperature increases suggests the presence of competing thermally 
activated non-radiative mechanisms, being most severe for 1@AA, compared with AA. Further arguments supporting this conclusion are found by the absolute emission quantum yield (q). Despite the low $q$ values measured for both samples, we note a larger one for AA (0.02 \pm 0.01 excited within 274-430 $\mathrm{nm}$ ) with respect to that found for $1 @ A$ A that lies below the detection limits of our experimental set-up $(<0.01)$.

The lifetime thermal dependence was rationalised using the classical Mott-Seitz model. ${ }^{28-29}$ In short, the excited state lifetime is given by $\tau(T)=\tau_{0} /\left(1+\alpha \exp \left(-\Delta E / k_{\mathrm{B}} T\right)\right.$, where $\tau_{0}$ is the excited state lifetime value at $T=0 \mathrm{~K}, \alpha$ the ratio between the non-radiative and radiative probabilities of the deactivation channel at $T=0 \mathrm{~K}$, and $\Delta E$ the activation energy of the thermal quenching process. From the data best fit (Fig. S22), similar $\Delta E$ and $\alpha$ values were found for AA/1@AA (804 $\pm 98 / 758 \pm 72$ $\mathrm{cm}^{-1}$ and $41 \pm 14 / 48 \pm 17$, respectively). The obtained $\Delta E$ values are in good agreement with the experimental energy difference between the high-wavelength component in the excitation spectra and the low-lying singlet emitting state (Fig. S13). In our experimental conditions, no noticeable changes in the photoluminescence features were detected below the magnetic ordering temperature.

In conclusion, we have shown that magnetic PBA nanoparticles could be easily post-functionalized by adsorption of an amino-luminophore. AA could be strongly adsorbed within the pores created by cyanometallate vacancies giving an exceptional thermal stability of the resulting functionalized nano-objects. In-depth analysis indicates a significant modification of the luminescence properties caused by the interaction between the AA luminophore and PBA framework while the long-range magnetic ordering is preserved affording bifunctional molecule-based nanomagnets. The proposed simple methodology could be extended to various functional guests and different PBA or others functional nano-objects in order to design multifunctional systems at the nanoscale.

\section{Conflicts of interest}

There are no conflicts of interest to declare.

\section{Acknowledgements}

The authors thank the University of Montpellier, CNRS and PAC of ICGM. The Portugal-France bilateral action, PESSOA Program (Hubert Curien) "Magneto-optical Multifunctional Nanoparticles" (40773PE) is also acknowledged. This work was developed within the scope of the project CICECO-Aveiro Institute of Materials, POCI-01-0145-FEDER-007679 (FCT Ref. UID/CTM/50011/2013) financed by national funds through the FCT/MEC and when appropriate co-financed by FEDER under the PT2020 Partnership Agreement. A.M.P. Botas thanks FCT for a PhD fellowship (SFRH/BD/104789/2014).

\section{Notes and references}

1 L. Ouahab, Multifunctional molecular materials, Pan Stanford, Singapore, 2013.

2 C. Train, T. Nuida, R. Gheorghe, M. Gruselle and S.-I. Ohkoshi, J. Am. Chem. Soc., 2009, 131, 16838-16843.

3 E. Coronado and P. Day, Chem. Rev., 2004, 104, 5419-5448.
4 P. Dechambenoit and J. R. Long, Chem. Soc. Rev., 2011, 40, 3249-3265.

5 S.-i. Ohkoshi, H. Tokoro, T. Matsuda, H. Takahashi, H. Irie and K. Hashimoto, Angew. Chem., Int. Ed., 2007, 46, 3238-3241.

6 E. Pardo, C. Train, H. Liu, L.-M. Chamoreau, B. Dkhil, K. Boubekeur, F. Lloret, K. Nakatani, H. Tokoro, S.-i. Ohkoshi and M. Verdaguer, Angew. Chem., Int. Ed., 2012, 51, 8356-8360.

7 F. Pointillart, O. Cador, B. Le Guennic and L. Ouahab, Coord. Chem. Rev., 2017, 346, 150-175.

8 J. Long, Y. Guari, R. A. S. Ferreira, L. D. Carlos and J. Larionova, Coord. Chem. Rev., 2018, 363, 57-70.

9 K. Kumar, S. Chorazy, K. Nakabayashi, H. Sato, B. Sieklucka and S.-i. Ohkoshi, J. Mater. Chem. C, 2018, 6, 8372-8384.

10 A. M. Spokoyny, D. Kim, A. Sumrein and C. A. Mirkin, Chem. Soc. Rev., 2009, 38, 1218-1227.

11 P. Horcajada, R. Gref, T. Baati, P. K. Allan, G. Maurin, P. Couvreur, G. Férey, R. E. Morris and C. Serre, Chem. Rev., 2012, $112,1232-1268$.

12 L. Catala and T. Mallah, Coord. Chem. Rev., 2017, 346, 32-61.

13 J. Long, Y. Guari, C. Guerin and J. Larionova, Dalton Trans., 2016, 45, 17581-17587.

14 M. F. Dumont, S. Yadavilli, R. W. Sze, J. Nazarian and R. Fernandes, Int J Nanomedicine, 2014, 9, 2581-2595.

15 S. Ye, Y. Liu, S. Chen, S. Liang, R. McHale, N. Ghasdian, Y. Lu and X. Wang, Chem. Commun., 2011, 47, 6831-6833.

16 J. Rocha, L. D. Carlos, F. A. A. Paz and D. Ananias, Chem. Soc. Rev., 2011, 40, 926-940.

17 Y. Cui, Y. Yue, G. Qian and B. Chen, Chem. Rev., 2012, 112, 11261162.

18 W. P. Lustig, S. Mukherjee, N. D. Rudd, A. V. Desai, J. Li and S. K. Ghosh, Chem. Soc. Rev., 2017, 46, 3242-3285.

19 Y. Zhang, S. Yuan, G. Day, X. Wang, X. Yang and H.-C. Zhou, Coord. Chem. Rev., 2018, 354, 28-45.

20 Q. Yao, A. Bermejo Gómez, J. Su, V. Pascanu, Y. Yun, H. Zheng, H. Chen, L. Liu, H. N. Abdelhamid, B. Martín-Matute and X. Zou, Chem. Mater., 2015, 27, 5332-5339.

21 G. Felix, W. Nicolazzi, L. Salmon, G. Molnar, M. Perrier, G. Maurin, J. Larionova, J. Long, Y. Guari and A. Bousseksou, Phys. Rev. Lett., 2013, 110, 235701/235701-235701/235705.

22 A. Takahashi, H. Tanaka, K. Minami, K. Noda, M. Ishizaki, M. Kurihara, H. Ogawa and T. Kawamoto, RSC Adv., 2018, 8, 3480834816.

23 Y. Prado, S. Mazerat, E. Rivière, G. Rogez, A. Gloter, O. Stéphan, L. Catala and T. Mallah, Adv. Funct. Mater., 2014, 24, 54025411.

24 V. Gadet, T. Mallah, I. Castro, M. Verdaguer and P. Veillet, J. Am. Chem. Soc., 1992, 114, 9213-9214.

25 Y. Uchiyama, R. Watanabe, T. Kurotaki, S. Kuniya, S. Kimura, Y. Sawamura, T. Ohtsuki, Y. Kikuchi, H. Matsuzawa, K. Uchiyama, M. Itakura, F. Kawakami and H. Maruyama, ACS Omega, 2017, 2, 3371-3379.

26 S. S. Thomas and C. Bohne, Faraday Discuss., 2015, 185, $381-$ 398.

27 K. Rotkiewicz and Z. R. Grabowski, Trans. Faraday Soc., 1969, 65, 3263-3278.

28 F. Seitz, Trans. Faraday Soc., 1939, 35, 74-85.

29 J. C. G. Bunzli and V. K. Pecharsky, Handbook on the Physics and Chemistry of Rare Earths: Including Actinides, Elsevier Science, 2016. 